\title{
Influence of Underlayer on Growth of Carbon Nanotubes Filled with Iron Nanowires*
}

\author{
Nobuo Kubonaka and Hideki Sato ${ }^{\dagger}$ \\ Department of Electrical and Electronic Engineering, \\ Graduate School of Engineering, Mie University, \\ 1577 Kurima-machiya-cho, Tsu, Mie, 514-8507, Japan
}

\section{Yuji Fujiwara}

Department of Physics Engineering, Graduate School of Engineering,

Mie University, 1577 Kurima-machiya-cho, Tsu, Mie, 514-8507, Japan

(Received 14 January 2015; Accepted 16 April 2015; Published 13 June 2015)

\begin{abstract}
Carbon nanotubes filled with iron nanowires (Fe@CNTs) show magnetic anisotropy because of the high aspect ratio of the nanowires and are expected to find numerous applications such as magnetic recording media, probes for scanning force microscopy and medical treatment for cancer (hyperthermia). These applications require precise control of Fe@CNT growth. This growth depends on the condition of the substrate surface on which the CNTs are grown. In this study, Fe@CNTs were synthesized by thermal chemical vapor deposition on various thin metal films (Fe, Ni, Co, Al, Pt, $\mathrm{Ti}$, or $\mathrm{Mo}$ ) deposited on $\mathrm{SiO}_{2} / \mathrm{Si}(100)$ substrates using ferrocene as a precursor, and their growth characteristics were investigated. The growth clearly depends on the metal species incorporated in the thin films. Magnetic properties correlated with growth morphologies. Therefore, the appropriate selection of an underlayer is important for controlling magnetic characteristics of Fe@CNTs. [DOI: 10.1380/ejssnt.2015.295]
\end{abstract}

Keywords: Carbon Nanotube; Iron Nanowires and Nanotubes; Magnetic Properties; Coercivity; Chemical Vapor Deposition

\section{INTRODUCTION}

Carbon nanotubes filled with iron nanowires (Fe@CNTs) [1-3] show high coercivity (typically $79.6 \mathrm{kA} / \mathrm{m}$ ) because of shape anisotropy originating from the high aspect ratio of the nanowires. In addition, their graphite layer serves as a protective layer for the encapsulated metal, thereby providing anticorrosivity to the nanowires. These properties make Fe@CNTs promising candidates for various applications, such as magnetic recording media $[4,5]$, medical treatment for cancer (hyperthermia) [6-8], and flexible magnetic devices, which require controlled growth and enhanced magnetic properties.

Several studies have addressed Fe@CNTs synthesized by thermal chemical vapor deposition (T-CVD), along with their growth characteristics and magnetic properties [9-21]. In our previous study, the Fe@CNT growth morphology strongly relied on growth temperature and the amount of precursor used in the T-CVD reactor and impacted magnetic properties; the coercivity exceeded $127.6 \mathrm{kA} / \mathrm{m}(1.6 \mathrm{kOe})$ because of the crystal structure of the Fe@CNT, which is remarkably valuable considering that iron is a soft magnetic material [22].

A growth mechanism has been proposed by Müller et al. for Fe@CNTs [18]. In this mechanism, Fe nanoparticles formed by the granulation of a previously deposited $\mathrm{Fe}$ film on the substrate act as growth sites. This mechanism essentially resembles that of conventional CNTs, except for the Fe nanowire encapsulation. The Fe@CNT growth mechanism plays a central role in growth control; however, the mechanism remains unclear.

We have recently found that the the T-CVD growth of Fe@CNTs shows selectivity toward metal species of

\footnotetext{
* This paper was presented at the 7th International Symposium on Surface Science, Shimane Prefectural Convention Center $(\mathrm{Ku}$ nibiki Messe), Matsue, Japan, November 2-6, 2014.

$\dagger$ Corresponding author: sato@elec.mie-u.ac.jp
}

underlayers, thin films deposited on the substrates before CNT growth. This selectivity may relate to metal nanoparticle formation. Therefore, a comprehensive examination of this phenomenon may clarify Fe@CNT growth characteristics and enable growth control. To this end, in this study, the influence of underlayer metal species on Fe@CNT growth was evaluated. Some metal species promoted growth, whereas others inhibited it. Relations among growth yield, CNT dimensions, and magnetic properties were investigated in detail.

\section{EXPERIMENTAL}

Silicon (Si) (100) single-crystal wafers $(10 \mathrm{~mm} \times$ $10 \mathrm{~mm} \times 0.5 \mathrm{~mm}$ ) coated with a $300-\mathrm{nm}$ thermal oxide layer were used as substrates for CNT growth. These substrates were cleaned in acetone and methanol by ultrasonication. Next, 2.0-nm-thick underlayers were deposited on substrates by vacuum evaporation ( $\mathrm{Fe}, \mathrm{Ni}, \mathrm{Co}$, $\mathrm{Al}$, or $\mathrm{Ti}$ ) or sputtering (Pt or $\mathrm{Mo}$ ). A substrate without the underlayer was also prepared as a control sample.

CNT growth was performed using a hot-wall CVD reactor [22,23] comprising a gas introduction system for carrier $\mathrm{Ar}$ gas and sublimated ferrocene $\left[\left(\mathrm{C}_{5} \mathrm{H}_{5}\right)_{2} \mathrm{Fe}\right]$ vapor, a quartz tube reactor heated using an electric furnace, and a mechanical rotary pump. The ferrocene precursor was heated to $150^{\circ} \mathrm{C}$ in the reservoir to promote sublimation. During the CVD process, each substrate was placed in the reactor, which was subsequently evacuated to approximately $2 \mathrm{~Pa}$ before introducing $\mathrm{Ar}$ at atmospheric pressure. Next, the reactor was heated to $785^{\circ} \mathrm{C}$ under Ar flow at $120 \mathrm{sccm}$, and ferrocene was introduced into the pre-heated reactor to conduct CNT growth for $10 \mathrm{~min}$. Finally, the influx of ferrocene was stopped, and the reactor was allowed to cool to $80^{\circ} \mathrm{C}$ under Ar flow before substrate removal.

Carbon nanotube growth morphologies were examined on the substrates by scanning electron microscopy (SEM). 

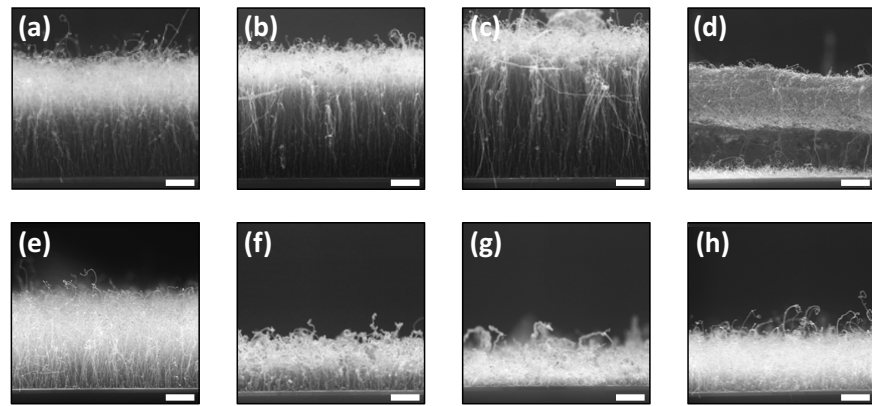

FIG. 1. Cross-sectional SEM images of CNTs grown on different substrates. Films grown on (a) the bare substrate and on (b) Fe, (c) Ni, (d) Co, (e) Al, (f) Pt, (g) Ti and (h) Mo underlayers. Scale bars are $2 \mu \mathrm{m}$.

Detailed CNT and encapsulated Fe nanowire dimensions were evaluated by transmission electron microscopy (TEM). Iron nanowire crystal structures were determined by selected area transmission electron diffraction (SAED). Magnetic properties were characterized using a vibrating sample magnetometer (VSM).

\section{RESULTS}

Figure 1 shows representative cross-sectional SEM images of CNTs grown by T-CVD on $\mathrm{SiO}_{2} / \mathrm{Si}(100)$ substrates (a) without underlayer (the control sample) and covered with various metal films including (b) Fe, (c) Ni, (d) Co, (e) Al, (f) Pt, (g) Ti and (h) Mo. Vertically oriented CNTs formed on $\mathrm{Fe}, \mathrm{Ni}$, and $\mathrm{Al}$ underlayers. Interestingly, the substrate without underlayer (i.e., $\mathrm{SiO}_{2}$ surface) also gave vertically oriented growth. On the other hand, Pt, Ti, and Mo underlayers generated randomly oriented CNTs. The CNT film peeled off the Co underlayer and exhibited uneven thickness despite a partial vertical alignment. In addition, the CNT film was covered by an aggregate layer that potentially consisted of amorphous carbon.

Film thicknesses were measured as average distances from substrate surfaces to CNT tips using the cross-

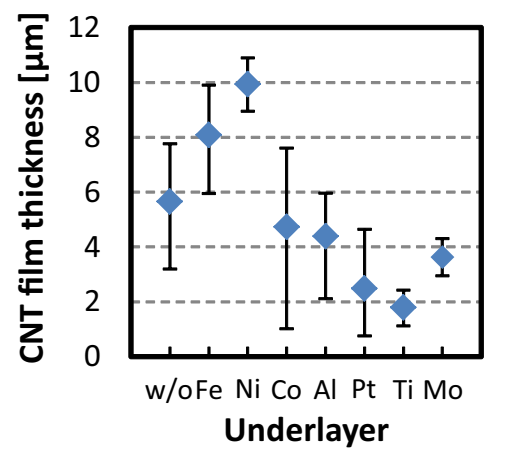

FIG. 2. CNT film thickness as a function of underlayer metal species.
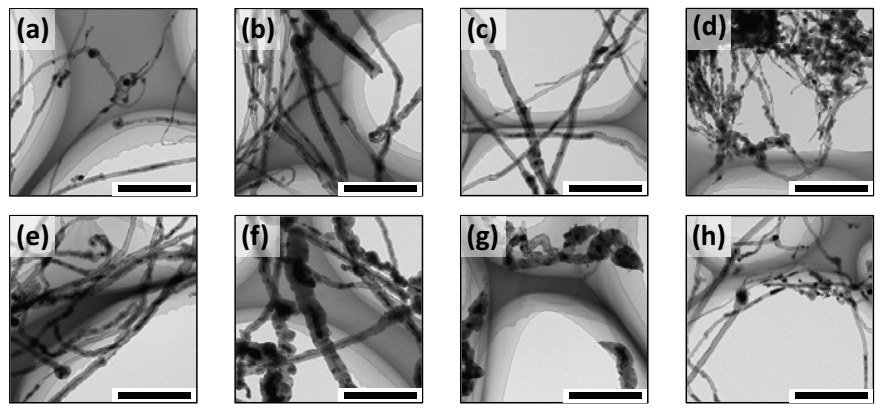

FIG. 3. TEM images of CNTs grown on different substrates. Films grown on (a) the bare substrate and on (b) Fe, (c) Ni, (d) Co, (e) Al, (f) Pt, (g) Ti and (h) Mo underlayers. Scale bars are $500 \mathrm{~nm}$.

sectional SEM images. Figure 2 shows the dependence of CNT film thickness on the nature of the underlayer. The nickel underlayer produced the thickest (about $10 \mu \mathrm{m}$ ) CNT film while the bare substrate gave the third thickest one. Because the CNT film formed on the Co underlayer partly peeled off, the thickness shown here corresponds to the intact part of the film.

The detailed growth morphology of the individual CNTs was examined by TEM. TEM images confirmed that metal nanowires were encapsulated in CNTs grown on substrates covered with $\mathrm{Fe}, \mathrm{Ni}, \mathrm{Al}, \mathrm{Pt}$, and Mo underlayers as well as without underlayer (Fig. 3). In contrast, samples generated on Co and Ti underlayers presented non-uniform metal encapsulation. These samples mostly consisted of granular encapsulated metal particles in addition to few wire-like metal objects. TEM images of CNTs grown on the Ti underlayer (Fig. 3g) displayed turbostratic fibers instead of tubular structures.

Nanotube and nanowire dimensions determined from
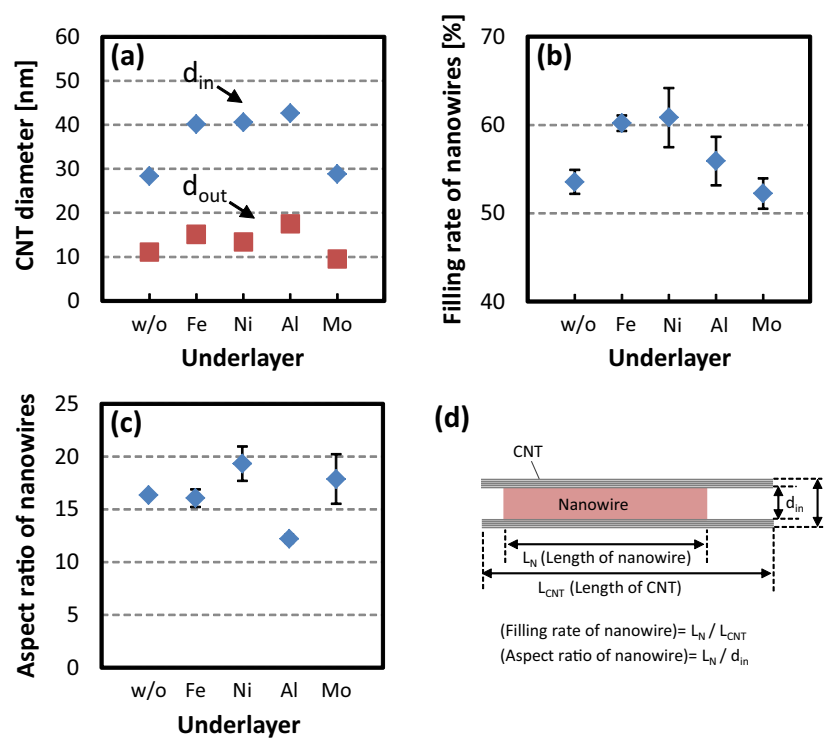

(d)

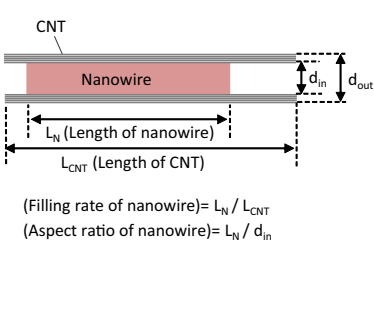

FIG. 4. (a) CNT diameters, (b) nanowire filling rates, and (c) aspect ratio as a function of underlayer metal species. (d) Definitions of the Fe@CNT filling rate and aspect ratio. 
TEM images were analyzed in detail. Figure 4a-c shows CNT outer and inner diameters, nanowire filling rates and aspect ratios. The filling rate indicates the volume ratio of Fe nanowire to the CNT inner cavity (as a percentage). Here, samples clearly presenting nanowire encapsulated inside CNTs by TEM (Fig. 3) were examined [i.e., with $\mathrm{Fe}, \mathrm{Ni}, \mathrm{Al}$, and Mo underlayers and without underlayer (w/o)]. Samples generated on Pt and Ti underlayers were neglected because of their poor morphologies. Dimensions correspond to averages of approximately 100 different randomly selected nanowires. Nanotubes formed on Mo-covered and bare substrates tended to display smaller outer $\left(\mathrm{d}_{\text {out }}\right)$ and inner diameters $\left(\mathrm{d}_{\mathrm{in}}\right)$ compared to other samples. Inner hollow diameters were almost constant for individual CNTs. Thus, filling rates were obtained by calculating $\mathrm{L}_{\mathrm{N}} / \mathrm{L}_{\mathrm{CNT}}$, where $\mathrm{L}_{\mathrm{N}}$ and $\mathrm{L}_{\mathrm{CNT}}$ are Fe nanowire and CNT lengths, respectively (Fig. 4d). Filling rates ranged between 50 and $65 \%$ while aspect ratios exceeded 10 for all samples.

These results indicate that the nature of the underlayer remarkably influences CNT growth. In general, CNT growth by CVD rests on catalyst nanoparticles serving as growth sites [24], and CNT growth assessed in this study is expected to follow this regime. To evaluate this hypothesis, surface conditions of underlayer-covered substrates were observed by SEM just before the onset of CNT growth. These substrates were ramp-heated under the same conditions as for CNT growth but heating was stopped when the CNT growth temperature was reached $\left(785^{\circ} \mathrm{C}\right)$. No ferrocene was introduced before the substrates were cooled and removed from the reactor. Figure 5 shows morphological SEM images of these underlayers. Substrates covered with Fe, Ni, Al, Pt, and Mo films presented granular particles, which may have formed by heat-induced granulation of the underlayer. Particle densities remarkably changed between samples. Dense particles were detected on $\mathrm{Ni}$ and $\mathrm{Pt}$ covered surfaces while Fe and $\mathrm{Al}$ films showed sparse particle formation. No clear granulation was observed by SEM for Ti films.

The crystal structure of the CNT-encapsulated nanowires was determined by SAED. Nanotubes grown without underlayer and with $\mathrm{Fe}, \mathrm{Ni}$ and $\mathrm{Al}$ underlayers, which showed relatively higher growth yields by SEM (Fig. 1), were examined (Fig. 6). All samples exhibited
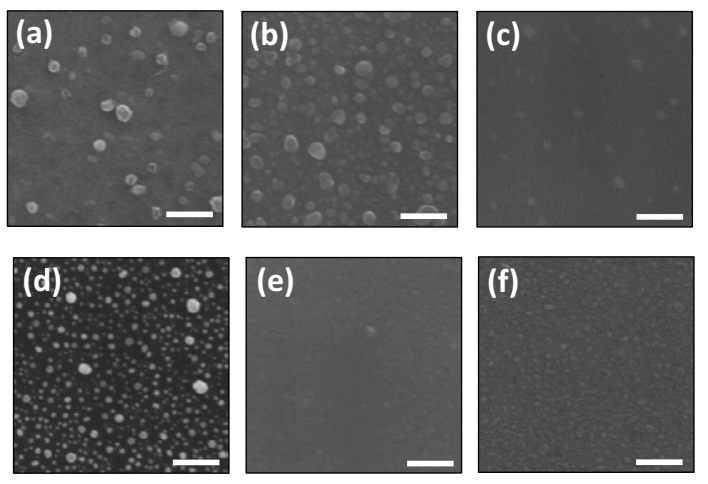

FIG. 5. SEM images of (a) Fe, (b) Ni, (c) Al, (d) Pt, (e) Ti, and (f) Mo underlayers deposited on the substrates after the heating. Scale bars are $200 \mathrm{~nm}$. diffraction rings attributable to $\alpha$-Fe and iron carbide $\left(\mathrm{Fe}_{3} \mathrm{C}\right)$, indicating that these compounds were encapsulated in the CNTs. The diffraction ring corresponding to $\alpha-\mathrm{Fe}(200)$ appeared clearly in the SAED pattern of CNTs grown on a Fe underlayer. Despite its lower intensity, this ring was also observed for CNTs grown on a $\mathrm{Ni}$ underlayer. In contrast, it was faint for samples grown without and on an $\mathrm{Al}$ underlayer, which mainly displayed the ring for $\mathrm{Fe}_{3} \mathrm{C}$. No other diffraction ring indicating the formation of alloys, such as $\mathrm{Fe}-\mathrm{Ni}$ and $\mathrm{Fe}-\mathrm{Al}$, was detected in these SAED patterns.

Magnetization curves of Fe@CNT films were measured by VSM to assess their magnetic properties. The normalized magnetization was plotted as a function of external magnetic field along parallel and perpendicular directions to the substrates (Fig. 7). The perpendicular direction corresponds to the CNT growth direction. Films grown on $\mathrm{Fe}$ and $\mathrm{Ni}$ underlayers showed clear differences in coercivity between vertical and parallel directions. These differences were also found in samples on bare or $\mathrm{Al}$ and Pt covered substrates, albeit to a smaller extent. Interestingly, the Pt underlayer led to relatively high coercivity despite a poor CNT growth.

Figure 8 shows the resulting perpendicular coercivities. Films grown on $\mathrm{Fe}$ and $\mathrm{Ni}$ underlayers exhibited the highest $(1.4 \mathrm{kOe})$ and second highest perpendicular coercivities $(1.2 \mathrm{kOe})$, respectively. Interestingly, the bare substrate gave the fourth largest coercivity, close to the third largest coercivity obtained using the Al covered substrate. These results agree with the dependence of the CNT film thickness on the underlayer metal species (Fig. 2) except for Co. Therefore, thicker CNT films tended to show larger perpendicular coercivities.

\section{DISCUSSION}

This study shows that Fe@CNT growth strongly depends on the underlayer metal species. These metal species may be divided into those promoting highyield $\mathrm{Fe} @ \mathrm{CNT}$ growth ( $\mathrm{Fe}, \mathrm{Ni}$ ), those leading to lowyield Fe@CNT growth (Al, Mo), and those giving poor Fe@CNT growth or turbostratic carbon structures (Co, $\mathrm{Pt}, \mathrm{Ti})$.

The growth model proposed by Deck et al. [25] is applicable to this Fe@CNT growth. This model postulates the formation of metal nanoparticles acting as growth sites on the substrate, catalyzing this CNT growth. On the other hand, ferrocene thermal decomposition forms $\mathrm{Fe}$ atoms, which are supplied to the CNT open tips, leading to $\mathrm{Fe}$ nanowire encapsulation. Here the formation of metal nanoparticles on the substrate plays an important role in Fe@CNT growth.

Iron and $\mathrm{Ni}$ underlayers usually serve as catalysts for CNT growth [24]. These films displayed extensive granulation on the substrates when the temperature increased to $785^{\circ} \mathrm{C}$ (Fig. 5a and b). This granulation enhances the catalytic activity of the films, promoting Fe@CNT growth. Nanotube films grown on $\mathrm{Fe}$ and $\mathrm{Ni}$ underlayers showed high thicknesses, consistent with their granulation-enhanced catalytic activities. These films also displayed different thicknesses, which may originate from dissimilar nanoparticle densities. The Ni underlayer 


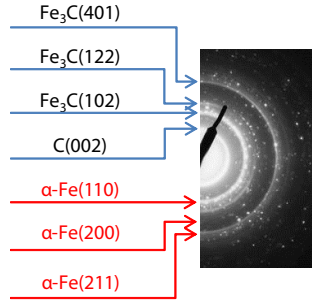

(a)
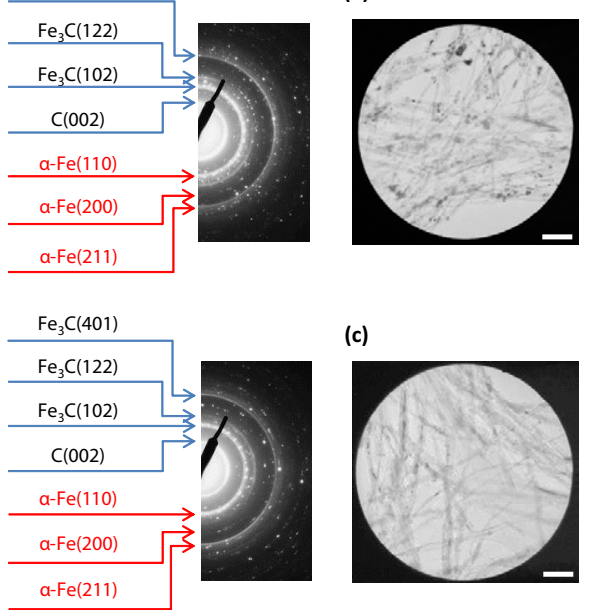

(c)

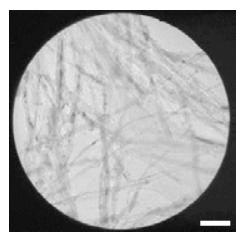

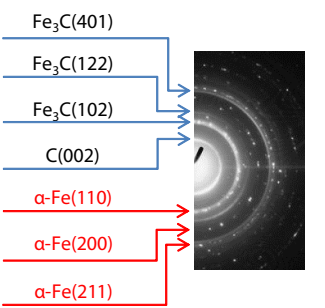

(b)

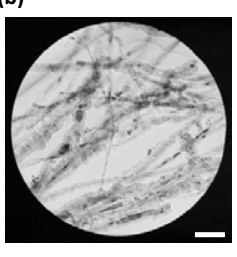

(d)

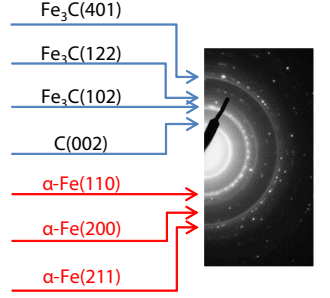

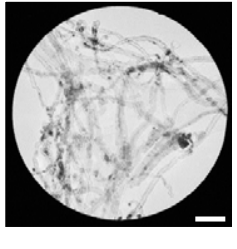

FIG. 6. SAED patterns and corresponding TEM images of Fe@CNTs. CNTs grown on (a) the bare substrate and on (b) Fe, (c) $\mathrm{Ni}$ and (d) Al underlayers. Scale bars are $250 \mathrm{~nm}$.
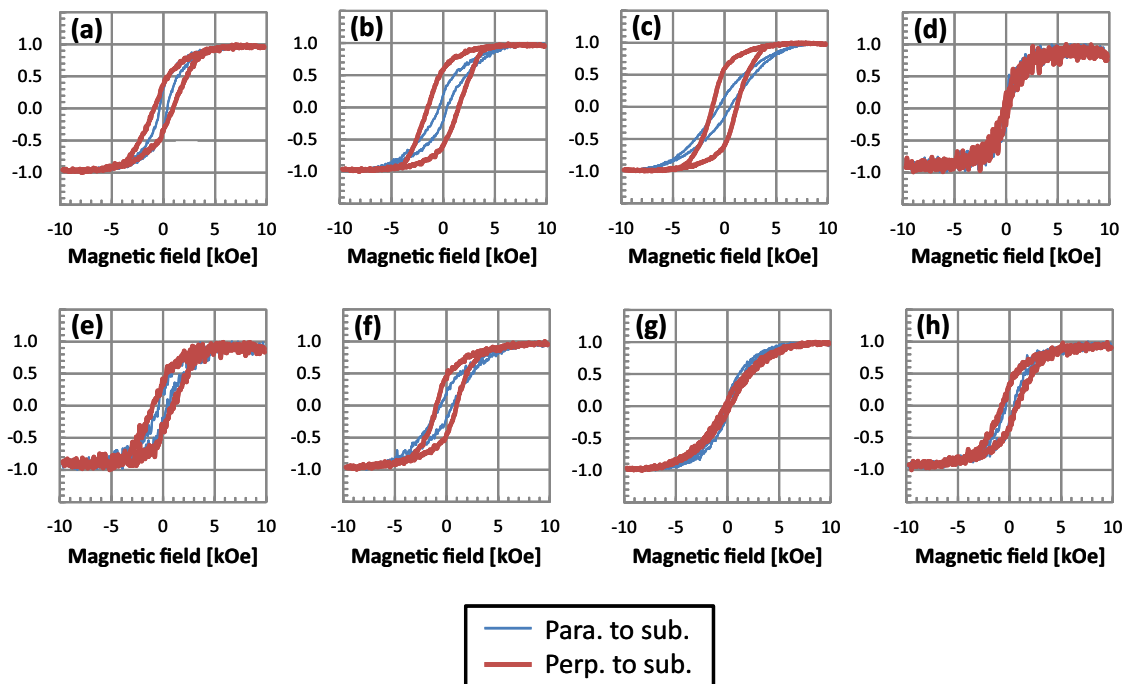

FIG. 7. Magnetization curves of Fe@CNTs grown on different substrates. (a) CNTs grown on the bare substrate and on (b) $\mathrm{Fe}$, (c) Ni, (d) Co, (e) Al, (f) Pt, (g) Ti and (h) Mo underlayers.

presented denser nanoparticle formation than its Fe counterpart (Fig. 5b), indicative of its higher catalytic activity. Cobalt has also been utilized as a catalyst for CNT growth [25]. However, the Co underlayer gave heterogeneous CNT films in this study. These CNT films peeled off, indicating their poor adhesion to the substrate. This peeling off may occur by $\mathrm{Co}-\mathrm{Fe}-\mathrm{C}$ alloy layer formation under high stress. The poor morphology of these films implies that catalyst nanoparticles did not form properly on the Co underlayer.

The Fe@CNTs also grew on Al and Mo underlayers, albeit at lower growth yields than on Fe and Ni films. Typically, $\mathrm{Al}$ and Mo do not display catalytic activity toward CNT growth but serve as co-catalysts. Here, the catalyst needed for CNT growth originated from the thermal decomposition of the ferrocene growth precursor, facilitating Fe nucleation and CNT growth despite the absence of a catalytic underlayer on the substrate. This process also explains CNT growth on the bare $\mathrm{SiO}_{2} / \mathrm{Si}$ substrate.

Nanotubes grown on $\mathrm{Pt}$ and $\mathrm{Ti}$ underlayers presented extremely poor growth morphologies. Specifically, no tubular structure was observed on the Ti underlayer. Platinum also displays catalytic activity under certain conditions [26], which differed from the current experimental conditions. Here, these underlayers inhibit the nucleation of Fe supplied by the ferrocene decomposition.

The underlayer dependence of the coercivities (Fig. 8) roughly coincides with that of the CNT film thickness (Fig. 2). This may result from Fe@CNT alignment on the substrate. Fe@CNTs grown on Fe and Ni underlayers exhibited good vertical alignment on the substrates and were magnetized along this axial direction. Therefore, the alignment of CNT growth influences magnetic properties. The shape (i.e., aspect ratio) and crystal structure of the encapsulated Fe nanowires also affect these properties, especially coercivities [27]. However, Fe@CNTs grown 


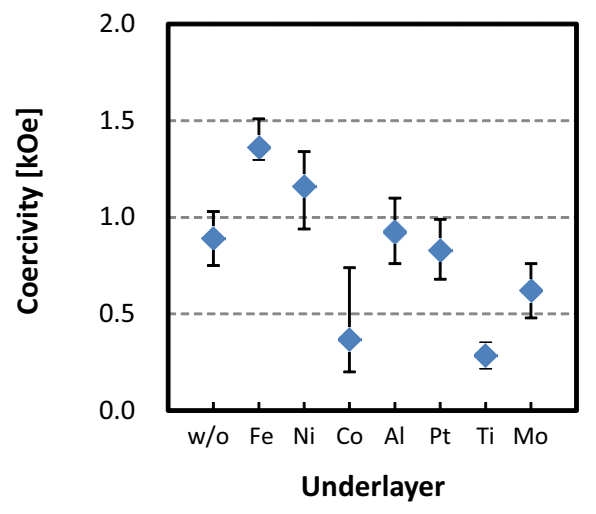

FIG. 8. Perpendicular coercivities of Fe@CNTs as a function of underlayer metal species.

on $\mathrm{Fe}, \mathrm{Ni}, \mathrm{Al}$, and $\mathrm{Mo}$ underlayers and bare substrate presented aspect ratios surpassing 10 (Fig. 4c), which is large enough for shape anisotropy [27]. Therefore, the effect of shape anisotropy on magnetic properties was neglected in this study. Magnetic properties also depend on Fe nanowire carburization. SAED patterns (Fig. 6) indicated that CNTs encapsulated $\alpha$-Fe and $\mathrm{Fe}_{3} \mathrm{C}$. The $\mathrm{Fe}_{3} \mathrm{C}$ encapsulation lowers the coercivity [28] whereas that of $\alpha$ Fe increases the coercivity. The SAED pattern of CNTs grown on the Fe underlayer showed a diffraction ring for $\alpha-\mathrm{Fe}(200)$. This ring was also found for CNTs grown on the $\mathrm{Ni}$ underlayer, albeit at lower intensity than on the $\mathrm{Fe}$ underlayer. Therefore, CNTs grown on Fe and Ni underlayers have higher coercivities than on other substrates as a result of $\alpha$-Fe encapsulation. A lower coercivity may stem from a shortage of Fe atoms on the substrate surface. Moreover, substrates covered with $\mathrm{Fe}$ and $\mathrm{Ni}$ underlayers exhibit catalytic activity. Consequently, Fe atoms produced by ferrocene decomposition are not consumed during catalyst film formation, leaving enough Fe atoms on the substrates. These atoms are mainly used to generate $\alpha$-Fe nanowires, which efficiently form in the CNT cavities. In contrast, non-catalytic underlayers, such as $\mathrm{Al}$ and the bare substrate, require the formation of catalytic films or nanoparticles on their surfaces during the first stages of CNT growth. Under these circumstances, many $\mathrm{Fe}$ atoms are consumed to produce catalytic nanoparticles, decreasing their amount compared with that of the carbon atoms. As a result, $\mathrm{Fe}_{3} \mathrm{C}$ forms preferentially. This mechanism agrees with the decrease in the nanowire filling rates of CNTs grown on non-catalytic underlayers (Fig. 4b).

CNT growth on non-Fe metal underlayers may also involve incorporation of these metals into Fe nanowires. No SAED diffraction pattern showed such an incorporation but this possibility cannot be ruled out. The crystallinity of the CNT-encapsulated nanowires may explain the absence of this phenomenon. Growth conditions may influence lattice defect formation, multigrain crystal structure formation, and crystal orientation changes in each nanowire, affecting magnetic properties. These speculations need further investigation to clarify the influence of underlayer components on Fe@CNT magnetic properties. Detailed examinations are in progress to clarify these effects.

\section{CONCLUSION}

The effect of the underlayer on Fe@CNT growth by T-CVD using ferrocene as a precursor was investigated. The Fe and Ni underlayers gave higher Fe@CNT growth yields than the other metals used in this study, consistent with their efficient granulation and the resulting higher nanoparticles catalytic activities. In contrast, $\mathrm{Ti}$ and Pt underlayers suppressed the Fe@CNT growth as a result of the inhibited catalyst nanoparticle formation. The Fe@CNTs grown on Fe and Ni underlayers showed high coercivities, which originated from $\alpha$-Fe nanowire production. The Fe nanowire crystal structure depended on the $\mathrm{Fe} / \mathrm{C}$ ratio on the substrate during Fe@CNT growth. Specifically, higher Fe concentrations led to $\alpha$ Fe nanowires. The Fe@CNTs also grew on $\mathrm{Al}$ and Mo underlayers as well as on the bare $\mathrm{SiO}_{2}$ surface. However, their growth yields and coercivities were lower than those of Fe@CNTs grown on Fe and Ni underlayers. Overall, underlayers significantly influence Fe@CNT growth morphologies and magnetic properties.

\section{ACKNOWLEDGMENTS}

This work was supported by a Japan Society for the Promotion of Science (KAKENHI No. 25390030). The authors would like to thank Prof. Y. Saito and Dr. K. Asaka, Nagoya University, as well as Prof. K. Iida, Mie University, for their help.
[1] C. N. R. Rao, R. Sen, B. C. Satishkumar, and A. Govindaraj, Chem. Commun. 15, 1525 (1998).

[2] A. Leonhardt, M. Ritschel, R. Kozhuharova, A. Graff, T. Mühl, R. Huhle, I. Mönch, D. Elefant, and C. M. Schneider, Diam. Relat. Mater. 12, 790 (2003).

[3] H. Nishino, C. Yamaguchi, H. Nakaoka, and R. Nishida, Carbon 41, 2165 (2003).

[4] C. P. Luo, S. H. Liou, and D. J. Sellmyer, J. Appl. Phys. 87, 6941 (2000).

[5] C. T. Kuo, C. H. Lin, and A. Y. Lo, Diam. Relat. Mater. 12, 799 (2003).
[6] I. Baker, Q. Zeng, W. D. Li, and C. R. Sullivan, J. Appl. Phys. 99, 08H106 (2006).

[7] Y. Krupskaya et al., J. Magn. Magn. Mater. 321, 4067 (2009).

[8] W. J. Atkinson, I. A. Brezovich, and D. P. Chakraborty, IEEE Trans. Biomed. Eng. 31, 70 (1984).

[9] N. Grobert et al., Appl. Phys. Lett. 75, 3363 (1999).

[10] S. Groudeva-Zotova, R. Kozhuharova, D. Elefant, T. Muhl, C. M. Schneider, and I. Monch, J. Magn. Magn. Mater. 306, 40 (2006). 
[11] C. X. Shi and H. T. Cong, J. Appl. Phys. 104, 034307 (2008).

[12] A. L. Elias et al., Nano Lett. 5, 467 (2005).

[13] R. Kozhuharova, M. Ritschel, D. Elefant, A. Graff, I. Monch, T. Muhl, C. M. Schneider, and A. Leonhardt, J. Magn. Magn. Mater. 290, 250 (2005).

[14] A. Leonhardt, M. Ritschel, D. Elefant, N. Mattern, K. Biedermann, S. Hampel, C. Muller, T. Gemming, and B. Buchner, J. Appl. Phys. 98, 074315 (2005).

[15] A. Leonhardt, S. Hampel, C. Muller, I. Monch, R. Koseva, M. Ritschel, D. Elefant, K. Biedermann, and B. Buchner, Chem. Vapor Depos. 12, 380 (2006).

[16] C. Müller, D. Elefant, A. Leonhardt, and B. Buchner, J. Appl. Phys. 103, 034302 (2008).

[17] N. Aguilo-Aguayo, J. L. Castano-Bernal, J. GarciaCespedes, and E. Bertran, Diam. Relat. Mater. 18, 953 (2009).
[18] C. Müller, A. Leonhardt, M. C. Kutz, B. Buchner, and H. Reuther, J. Phys. Chem. C 113, 2736 (2009).

[19] U. Weissker et al., J. Appl. Phys. 106, 054909 (2009).

[20] K. Lipert, S. Bahr, F. Wolny, P. Atkinson, U. Weissker, T. Muhl, O. G. Schmidt, B. Buchner, and R. Klingeler, Appl. Phys. Lett. 97, 212503 (2010).

[21] F. C. Dillon, A. Bajpai, A. Koos, S. Downes, Z. Aslam, and N. Grobert, Carbon 50, 3674 (2012).

[22] H. Sato, A. Nagata, N. Kubonaka, and Y. Fujiwara, Jpn. J. Appl. Phys. 52, 11NL03 (2013).

[23] A. Nagata, H. Sato, Y. Matsui, T. Kaneko, and Y. Fujiwara, Vacuum 87, 182 (2013).

[24] A-C. Dupris, Prog. Mater Sci. 50, 929 (2005).

[25] C. P. Deck and K. Vecchio, Carbon 43, 2608 (2005).

[26] D. Takagi, Y. Homma, H. Hibino, S. Suzuki, and Y. Kobayashi, Nano Lett. 6, 2642 (2006).

[27] R. M. Bozorth and D. M. Chapin, J. Appl. Phys. 13, 320 (1942).

[28] J. Qiu, Q. Li, Z. Wang, Y. Sun, and H. Zhang, Carbon 44, 2565 (2006). 